\title{
Optimal target search on a fast folding polymer chain with volume exchange
}

\author{
Michael A. Lomholt, Tobias Ambjörnsson, and Ralf Metzler \\ NORDITA, Blegdamsvej 17, 2100 Copenhagen Ø, Denmark
}

\begin{abstract}
We study the search process of a target on a rapidly folding polymer ('DNA') by an ensemble of particles ('proteins'), whose search combines 1D diffusion along the chain, Lévy type diffusion mediated by chain looping, and volume exchange. A rich behavior of the search process is obtained with respect to the physical parameters, in particular, for the optimal search.
\end{abstract}

PACS numbers: 05.40.Fb,02.50.-Ey,82.39.-k

Introduction. Lévy flights (LFs) are random walks whose jump lengths $x$ are distributed like $\lambda(x) \simeq|x|^{-1-\alpha}$ with exponent $0<\alpha<2[\underline{1}$. Their probability density to be at position $x$ at time $t$ has the characteristic function $P(q, t) \equiv \int_{-\infty}^{\infty} e^{i q x} P(x, t) d x=\exp \left(-D_{\mathrm{L}}|q|^{\alpha} t\right)$, a consequence of the generalized central limit theorem 2]; in that sense, LFs are a natural extension of normal Gaussian diffusion $(\alpha=2)$. LFs occur in a wide range of systems [3]; in particular, they represent an optimal search mechanism in contrast to locally oversampling Gaussian search [4]. Dynamically, LFs can be described by a space-fractional diffusion equation $\partial P / \partial t=$ $D_{\mathrm{L}} \partial^{\alpha} P(x, t) / \partial|x|^{\alpha}$, a convenient basis to introduce additional terms, as shown below. $D_{\mathrm{L}}$ is a diffusion constant of dimension $\mathrm{cm}^{\alpha} / \mathrm{sec}$, and the fractional derivative is defined via its Fourier transform, $\mathscr{F}\left\{\partial^{\alpha} P(x, t) / \partial|x|^{\alpha}\right\}=$ $-|q|^{\alpha} P(q, t)$ [3]. LFs exhibit superdiffusion in the sense that $\left\langle|x|^{\zeta}\right\rangle^{2 / \zeta} \simeq\left(D_{\mathrm{L}} t\right)^{2 / \alpha}(0<\zeta<\alpha)$, spreading faster than the linear dependence of standard diffusion $(\alpha=2)$.

A prime example of an LF is linear particle diffusion to next neighbor sites on a fast folding ('annealed') polymer that permits intersegmental jumps at chain contact points (see Fig. 1) due to polymer looping [5, 6]. The contour length $|x|$ stored in a loop between such contact points is distributed in 3D like $\lambda(x) \simeq|x|^{-1-\alpha}$, where $\alpha=1 / 2$ for Gaussian chains ( $\theta$ solvent), and $\alpha \approx 1.2$ for self-avoiding walk chains (good solvent) 7].

While non-specifically bound 8], proteins can diffusively slide along the DNA backbone in search of their specific target site, as long as the binding energy does not exceed a certain limit [9]. Under overstretching conditions preventing looping, pure 1D sliding search could be observed in vitro [10]. In absence of the stretching force, the combination of intersegmental jumps (LF component) and 1D sliding may be a good approximation to the motion of binding proteins or enzymes along a DNA. In general, however, proteins detach to the volume and, after a bulk excursion, reattach successively before reaching the target. This mediation by de- and (re)adsorption rates $k_{\text {off }}$ and $k_{\text {on }}$ is described by the Berg-von Hippel model sketched in Fig. 11 11. We here explore by combination of analytical and numerical analysis for the first time (1) the combination of 1D sliding, intersegmental transfer and volume exchange, (2) a particle number density instead of a single searching protein; and (3) the explicit determination of the first arrival to the target, per

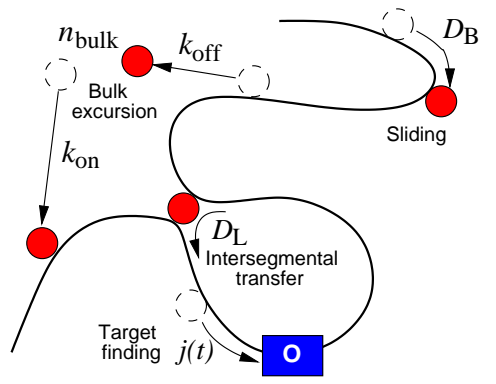

FIG. 1: Search mechanisms in Eq. (11).

se a non-trivial problem for LFs [12. Note that, although the process we study is a generic soft matter problem, we here adopt the DNA-protein language for illustration.

Theoretical description. In our description of the target search process, we use the density per length $n(x, t)$ of proteins on the DNA as the relevant dynamical quantity ( $x$ is the distance along the DNA contour). Apart from intersegmental transfer, we include 1D sliding along the DNA with diffusion constant $D_{\mathrm{B}}$, protein dissociation with rate $k_{\text {off }}$ and (re)adsorbtion with rate $k_{\text {on }}$ from a bath of proteins of concentration $n_{\text {bulk }}$. The dynamics of $n(x, t)$ is thus governed by the equation 13.

$$
\begin{aligned}
\frac{\partial}{\partial t} n(x, t)= & \left(D_{\mathrm{B}} \frac{\partial^{2}}{\partial x^{2}}+D_{\mathrm{L}} \frac{\partial^{\alpha}}{\partial|x|^{\alpha}}-k_{\text {off }}\right) n(x, t) \\
& +k_{\text {on }} n_{\text {bulk }}-j(t) \delta(x) .
\end{aligned}
$$

Here, $j(t)$ is the flux into the target located at $x=0$. We determine the flux $j(t)$ by assuming that the target is perfectly absorbing: $n(0, t)=0$. Be initially the system at equilibrium, except that the target is unoccupied; then, the initial protein density is $n_{0}=n(x, 0)=k_{\text {on }} n_{\text {bulk }} / k_{\text {off }}$ [14]. The total number of particles that have arrived at the target up to time $t$ is $J(t)=\int_{0}^{t} d t^{\prime} j\left(t^{\prime}\right)$. We derive explicit analytic expressions for $J(t)$ in different limiting regimes, and study the general case numerically. We use $J(t)$ to obtain the mean first arrival time $T$ to the target; in particular, to find the value of $k_{\text {off }}$ that minimizes $T$.

To proceed, we Laplace and Fourier transform Eq. (11):

$$
\begin{aligned}
& u n(q, u)-2 \pi n_{0} \delta(q)=-\left(D_{\mathrm{B}} q^{2}+D_{\mathrm{L}}|q|^{\alpha}+k_{\mathrm{off}}\right) \\
& \times n(q, u)+2 \pi k_{\mathrm{on}} n_{\mathrm{bulk}} \delta(q) / u-j(u),
\end{aligned}
$$

with $n(q, u)=\mathscr{L}\{n(q, t)\}$. Integration over $q$ produces 


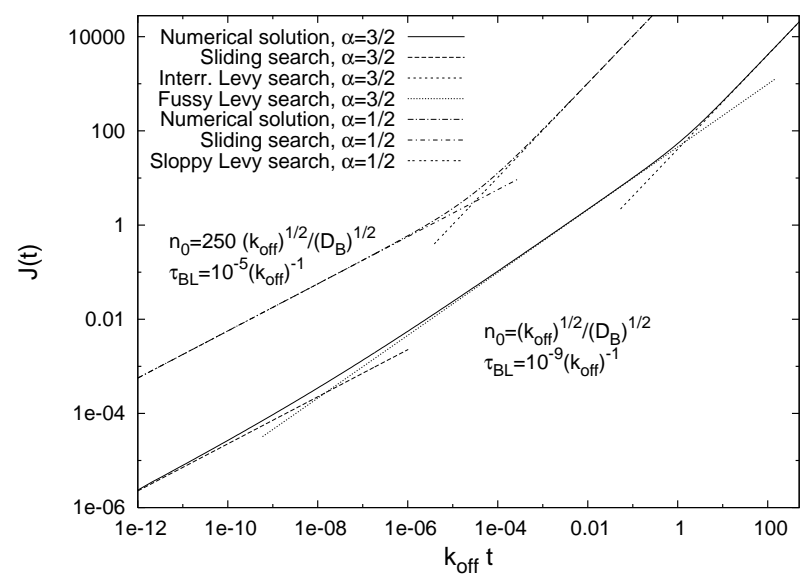

FIG. 2: Number of proteins arrived at the target up to $t$. Numerical solutions of Eq. (3) and limiting regimes.

$J(u)=j(u) / u=n_{0} /\left[u^{2} W_{0}(u)\right]$ due to the perfect absorption condition $n(0, u)=(2 \pi)^{-1} \int d q n(q, u)=0$. Or,

$$
\int_{0}^{t} d t^{\prime} W_{0}\left(t-t^{\prime}\right) J\left(t^{\prime}\right)=n_{0} t
$$

in the $t$-domain. Eq. (3) is a Volterra integral equation of the first kind, whose kernel $W_{0}$ is read off Eq. (2):

$$
W_{0}(u)=\int_{-\infty}^{\infty} \frac{d q}{2 \pi} \frac{1}{D_{\mathrm{B}} q^{2}+D_{\mathrm{L}}|q|^{\alpha}+k_{\mathrm{off}}+u},
$$

that is the Laplace transform of the Green's function of $n(x, t)$ at $x=0$. Back-transforming, we obtain $W_{0}(t)=(2 \pi)^{-1} \int_{-\infty}^{\infty} d q \exp \left(-\left(D_{\mathrm{B}} q^{2}+D_{\mathrm{L}}|q|^{\alpha}+k_{\mathrm{off}}\right) t\right)$, which has a singularity at $t=0$. Eq. (3) can be solved numerically by approximating $J(t)$ by a piecewise linear function, converting the integral equation to a linear set of equations. Typical plots are shown in Fig. 2

Eq. (4) reveals only two relevant time scales: $k_{\text {off }}^{-1}$ and $\tau_{\mathrm{BL}}=\left(D_{\mathrm{B}}^{\alpha} / D_{\mathrm{L}}^{2}\right)^{1 /(2-\alpha)}$. We now obtain asymptotic results for small and large $\left(k_{\text {off }}+u\right)$, compared to $\tau_{\mathrm{BL}}^{-1}$.

$k_{\mathrm{off}}+u \gg \tau_{\mathrm{BL}}^{-1}$ : In this limit, the denominator of the integrand in Eq. (4) is dominated either by the term $D_{\mathrm{B}} q^{2}$ or by $k_{\text {off }}+u$ for any $q$; we find the approximation [15]

$$
\left.W_{0}(u) \sim W_{0}(u)\right|_{D_{\mathrm{L}}=0}=\left[D_{\mathrm{B}}\left(k_{\mathrm{off}}+u\right)\right]^{-1 / 2} / 2 .
$$

$k_{\mathrm{off}}+u \ll \tau_{\mathrm{BL}}^{-1}$ and $\alpha>1$ ('connected LFs'): Here, a singularity exists at small $q$ as $k_{\text {off }}+u \rightarrow 0$. For finite but small $k_{\text {off }}+u \rightarrow 0$, the integrand is dominated by the $D_{\mathrm{L}}|q|^{\alpha}$ term compared to $D_{\mathrm{B}} q^{2}$ at small $q$, yielding

$$
W_{0}(u) \sim\left[\alpha \sin (\pi / \alpha) D_{\mathrm{L}}^{1 / \alpha}\left(k_{\text {off }}+u\right)^{1-1 / \alpha}\right]^{-1} .
$$

$k_{\text {off }}+u \ll \tau_{\mathrm{BL}}^{-1}$ and $\alpha<1$ ('disconnected LFs'): Now, the singularity is weak, and the integral becomes

$$
W_{0}(u) \sim\left[(2-\alpha) \sin \left(\frac{1-\alpha}{2-\alpha} \pi\right) \sqrt{D_{\mathrm{B}} \tau_{\mathrm{BL}}^{-1}}\right]^{-1} .
$$

From these limits, we now infer the behavior of $J(t)$, based on Tauberian theorems stating that $J(t)$ at $t \rightarrow 0$ is determined by $J(u)$ at $u \rightarrow \infty$, and vice versa [1]. We discover a rich variety of domains, compare Tab. [1

(1.) Sliding search: Desorption from the DNA can be neglected for times $t \ll k_{\text {off }}^{-1}$. If also $t \ll \tau_{\mathrm{BL}}$, Eq. (5) with $k_{\text {off }}=0$ by inverse Laplace transform leads to

$$
J(t) \sim\left(t / \tau_{1}\right)^{\gamma_{1}}, \gamma_{1}=1 / 2, \tau_{1}=\pi /\left(16 D_{B} n_{0}^{1 / \gamma_{1}}\right) .
$$

In this regime, only the $1 \mathrm{D}$ sliding mechanism matters.

(2.) Fussy Lévy search: For $\tau_{\mathrm{BL}} \ll t \ll k_{\mathrm{off}}^{-1}(\alpha>1)$, the LF dominates the flux into the target; from Eq. (6),

$$
J(t) \sim\left(t / \tau_{2}\right)^{\gamma_{2}}, \gamma_{2}=1 / \alpha, \tau_{2}=C_{2} /\left(D_{L} n_{0}^{1 / \gamma_{2}}\right),
$$

where $C_{2}=\{\Gamma(1+1 / \alpha) /[\alpha \sin (\pi / \alpha)]\}^{\alpha}$. Now, LFs are the overall dominating mechanism. This contrasts:

(3.) Sloppy Lévy search: For $\alpha<1, t \gg \tau_{\mathrm{BL}}$, and $k_{\text {off }}^{-1} \gg \tau_{\mathrm{BL}}$, we obtain from Eq. (7)

$$
J(t) \sim\left(\frac{t}{\tau_{3}}\right)^{\gamma_{3}}, \gamma_{3}=1, \tau_{3}=C_{3} \frac{D_{B}^{\alpha /[2(2-\alpha)]-1 / 2}}{D_{L}^{1 /(2-\alpha)} n_{0}^{1 / \gamma_{3}}},
$$

and $C_{3}=\{(2-\alpha) \sin ([1-\alpha] \pi /[2-\alpha])\}^{-1}$. For $\alpha<$ 1 , even the step length $\int d x|x| \lambda(x)$ diverges, making it impossible for the protein to hit a small target solely by LF, and local sampling by $1 \mathrm{D}$ sliding becomes vital. At longer times, volume exchange mediated by $k_{\text {off }}$ enters:

(4.) Interrupted Lévy search: For $\alpha>1$ and $t \gg$ $k_{\text {off }}^{-1} \gg \tau_{\text {BL }}$ we can ignore $u$ in Eq. ([6]), yielding

$$
J(t) \sim\left(t / \tau_{4}\right)^{\gamma_{4}}, \gamma_{4}=1, \tau_{4}=C_{4} /\left(D_{L}^{1 / \alpha} k_{\text {off }}^{1-1 / \alpha} n_{0}^{1 / \gamma_{4}}\right),
$$

with $C_{4}=1 /[\alpha \sin (\pi / \alpha)]$. The search on the DNA is dominated by LFs, interrupted by $3 \mathrm{D}$ volume excursions.

(5.) Interrupted sliding search: If $\tau_{\mathrm{BL}} \gg k_{\text {off }}^{-1}$, LFs will not contribute at any $t$. Instead we find from Eq. (5)

$$
J(t) \sim\left(t / \tau_{5}\right)^{\gamma_{5}}, \gamma_{5}=1, \tau_{5}=1 /\left(2 D_{B}^{1 / 2} k_{\text {off }}^{1 / 2} n_{0}^{1 / \gamma_{5}}\right)
$$

for $t \gg k_{\text {off }}^{-1}$. This is sliding-dominated search with 3D excursions. There exist three scaling regimes for $1<\alpha<$ 2, and two for $0<\alpha<1$; see Fig. [2] and Tab. [)

We found that the relevant time scales $k_{\text {off }}^{-1}$ and $\tau_{\mathrm{BL}}$ together with $\alpha$ give rise to 5 basic search regimes, each characterized by an exponent $\gamma_{i}$ and characteristic time scale $\tau_{i}$. In particular, we saw that $J(t) \sim\left(t / \tau_{i}\right)^{\gamma_{i}}$, where the exponent $\gamma_{i} \neq 1$ for the first two regimes $(i=1,2)$; in the other cases, we have $\gamma_{i}=1$. The stable index $\alpha$ characterizing the polymer statistics thus strongly influences the overall search. Also note that $J(t) \simeq t$ when $t \gg k_{\text {off }}^{-1}$, or $t \gg \tau_{\mathrm{BL}}$ and $\alpha<1$. The characteristic time scales $\tau_{i}$, since $J(t) \simeq n_{0}$, scale like $\tau_{i} \simeq n_{0}^{-1 / \gamma_{i}}$. As any integral $I=\int_{0}^{\infty} d t f\left(t / \tau_{i}\right)$ can be transformed by $s \equiv t / \tau_{i}$ to $I=\tau_{i} \int_{0}^{\infty} d s f(s)$, it is $I \simeq \tau_{i}$. Thus, we find that the mean first arrival time scales like 


\begin{tabular}{|l|c|c|c|}
\hline Regime & $0<\alpha<1$ & $1<\alpha<2$ & $J \sim\left(t / \tau_{i}\right)^{\gamma_{i}}$ \\
\hline$t \ll\left\{\tau_{\mathrm{BL}}, k_{\text {off }}^{-1}\right\}$ & Sliding & Sliding & $\gamma_{1}=1 / 2$ \\
$\tau_{\mathrm{BL}} \ll t \ll k_{\text {off }}^{-1}$ & Sloppy Lévy & Fussy Lévy & $\gamma_{3}=1 \mid \gamma_{2}=\alpha^{-1}$ \\
$\tau_{\mathrm{BL}} \ll k_{\text {off }}^{-1} \ll t$ & Sloppy Lévy & Int. Lévy & $\gamma_{3}=1 \mid \gamma_{4}=1$ \\
$\left\{t, \tau_{\mathrm{BL}}\right\} \gg k_{\text {off }}^{-1}$ & Int. Sliding & Int. Sliding & $\gamma_{5}=1$ \\
\hline
\end{tabular}

TABLE I: Summary of search regimes. See text.

$T=\tau_{i} \int_{0}^{\infty} d s \exp \left(-s^{\gamma_{i}}\right)=\tau_{i} \Gamma\left(1 / \gamma_{i}\right) / \gamma_{i} \simeq n_{0}^{-1 / \gamma_{i}}$ (see below) whenever a single of the five regimes dominates the integral. In particular, the variation of $T^{-1}$ with the line density $n_{0}$ ranges from quadratic (1D sliding) over $n_{0}^{\alpha}$ in the fussy Lévy regime $(1<\alpha<2)$ to linear, the latter being shared by sloppy Lévy and bulk mediated search. Note that if $1 \mathrm{D}$ sliding is the sole prevalent mechanism, we recover the result $T=\pi /\left[8 D_{\mathrm{B}} n_{0}^{2}\right]$ of Ref. 10].

Optimal search. We now address the optimal search of the target, i.e., which $k_{\text {off }}$ minimizes the mean first arrival time $T$ when $D_{\mathrm{B}}, D_{\mathrm{L}}, k_{\text {on }}$, the DNA length $L$, and the total amount of proteins are fixed. To quantify the latter, we define $l_{\text {DNA }} \equiv L / V$, where $V$ is the system volume. The overall protein volume density is then $n_{\text {total }}=l_{\mathrm{DNA}} n_{0}+n_{\text {bulk }}$. With the equilibrium condition $k_{\text {off }} n_{0}=k_{\text {on }} n_{\text {bulk }}$, this yields $n_{0}=n_{\text {total }} k_{\text {on }} /\left(k_{\text {off }}+k_{\text {on }}^{\prime}\right)$ and a corresponding expression for $n_{\text {bulk }}$; here, $k_{\text {on }}^{\prime}=$ $k_{\text {on }} l_{\text {DNA }}$ is the inverse average time a single protein spends in the bulk solvent before (re)binding to the DNA.

To extract the mean first arrival time $T$, we reason as follows (compare Ref. 10]): The total number of proteins that have arrived at the target between $t^{\prime}=0$ and $t$ is $J(t)$. If $N$ is the overall number of proteins, the probability for an individual protein to have arrived at the target is $J(t) / N$. In the limit of large $N$, we obtain the survival probability of the target (no protein has arrived) as

$$
P_{\text {surv }}(t)=\lim _{N \rightarrow \infty}(1-J(t) / N)^{N}=\exp [-J(t)],
$$

and thus $T=\int_{0}^{\infty} d t P_{\text {surv }}(t)$. Note that for LFs, the first arrival is crucially different from the first passage [12].

The optimization is complicated by the exponential function in Eq. (13). However, both in vitro and in vivo, $n_{\text {total }}$ (and hence $n_{0}$ ) is in many cases sufficiently small, such that the relevant regime is $J(t) \propto t$ (i.e., we can approximate $W_{0}(u)$ by $W_{0}(u=0)$ ). The mean first arrival time in this linear regime becomes

$$
T=W_{0}(u=0)\left[\left(k_{\text {off }}+k_{\text {on }}^{\prime}\right) / k_{\text {on }}^{\prime}\right]\left[l_{\text {DNA }} / n_{\text {total }}\right] .
$$

We observe a tradeoff in the optimal value $k_{\text {off }}^{\text {opt }}$, that minimizes $T$ : The fraction $k_{\text {on }}^{\prime} /\left(k_{\text {off }}+k_{\text {on }}^{\prime}\right)$ of bound proteins shrinks with increasing $k_{\text {off }}$, increasing $T$. Counteracting is the decrease of $W_{0}(u=0)$ (and $T$ ) with growing $k_{\text {off }}$.

Numerical solutions to the optimal search are shown in Fig. 3] for different $\alpha$. Three different regimes emerge:

(i) Without LFs $\left(D_{\mathrm{L}} \rightarrow 0\right.$ or $\left.D_{\mathrm{L}} \ll D_{\mathrm{B}}^{\alpha / 2}\left(k_{\mathrm{on}}^{\prime}\right)^{1-\alpha / 2}\right)$, from Eq. (5) with $W_{0}$ at $u=0$, we obtain $k_{\text {off }}^{\text {opt }}=k_{\text {on }}^{\prime}$ : the proteins should spend equal amounts of time in bulk

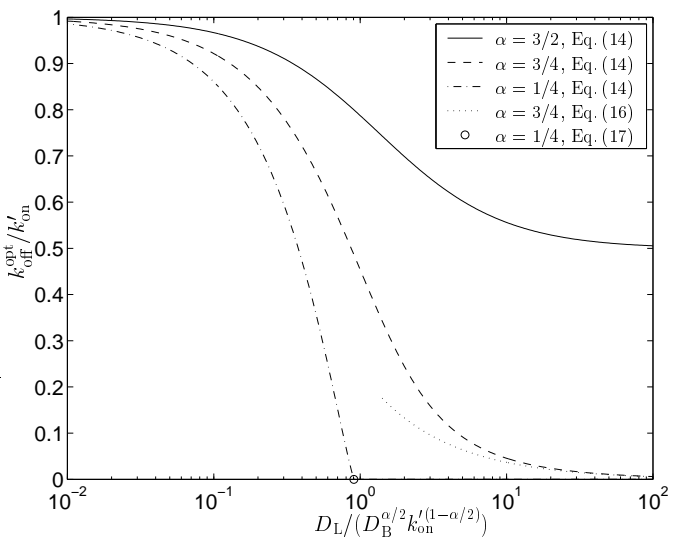

FIG. 3: Optimal choice of off rate $k_{\text {off }}$ as function of the LF diffusion constant, from numerical evaluation of Eq. (14). The

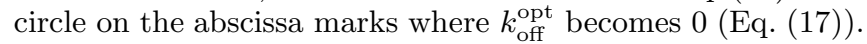

and on the DNA. This corresponds to the result obtained for single protein searching on a long DNA [9, 16]. Two additional regimes unfold for strong LF search, $D_{\mathrm{L}} \rightarrow \infty$ :

(ii) For $\alpha>1$, where Eq. (6) applies, we find

$$
k_{\mathrm{off}}^{\mathrm{opt}} \sim(\alpha-1) k_{\mathrm{on}}^{\prime}:
$$

The optimal off rate shrinks linearly with decreasing $\alpha$.

(iii) For $\alpha<1$, the value of $k_{\text {off }}^{\text {opt }}$ approaches zero as $D_{\mathrm{L}} \rightarrow \infty$ : The sloppy LF mechanism becomes so efficient that bulk excursions become irrelevant. More precisely, for $1 / 2<\alpha<1$ as $D_{\mathrm{L}}$ goes to infinity,

$$
k_{\mathrm{off}}^{\mathrm{opt}} \sim\left(\frac{(2-\alpha)(1-\alpha) \sin \left(\frac{1-\alpha}{2-\alpha} \pi\right)}{\alpha^{2} \sin \left(\frac{2 \alpha-1}{\alpha} \pi\right)} k_{\mathrm{on}}^{\prime} \tau_{\mathrm{BL}}^{1 / \alpha-1}\right)^{\frac{\alpha}{2 \alpha-1}}
$$

At $\alpha=1 / 2$, we observe a qualitative change: When $\alpha<$ $1 / 2$, the rate $k_{\mathrm{off}}^{\mathrm{opt}}$ reaches zero for all finite $D_{\mathrm{L}}$ satisfying

$$
\tau_{\mathrm{BL}}^{-1} \geq \frac{(1+\alpha) \sin ([1-\alpha] \pi /[2-\alpha])}{(2-\alpha) \sin ([1-2 \alpha] \pi /[2-\alpha])} k_{\mathrm{on}}^{\prime} .
$$

Note that when $\alpha<1$, the spread of the $\operatorname{LF}\left(\simeq t^{1 / \alpha}\right)$ grows faster than the number of sites visited $(\simeq t)$, rendering the mixing effect of bulk excursions insignificant. A scaling argument to understand the crossover at $\alpha=1 / 2$ relates the probability density of first arrival with the width $\left(\simeq t^{1 / \alpha}\right)$ of the Green's function of an $\mathrm{LF}, p_{\mathrm{fa}} \simeq t^{-1 / \alpha}$. The associated mean arrival time becomes finite for $0<\alpha<1 / 2$, even for the infinite chain considered here.

Discussion. Eq. (11) phrases the target search problem as a fractional diffusion-reaction equation with point sink. This formulation pays tribute to the fact that for LFs, the first arrival differs from the first passage: With the long-tailed $\lambda(x)$ of an LF, the particle can repeatedly jump across the target without hitting, the first arrival becoming less efficient than the first passage [12]. 
A borderline role is played by the Cauchy case $\alpha=1$, separating connected (mean jump length $\langle|x|\rangle$ exists) and disconnected LFs. For $\alpha<1$, the number of visited sites grows slower than the width of the search region and the LF mimics the uncorrelated jumps of bulk excursion; the latter becomes obsolete for high LF diffusivity $D_{\mathrm{L}}$. Below $\alpha=1 / 2$, bulk excursions already for finite $D_{\mathrm{L}}$ are undesirable. A similar observation can be made for the scaling of the mean search time $T$ with the Lévy diffusivity $D_{\mathrm{L}}$, that is proportional to the rate an LF is performed: For $\alpha>1$ in the interrupted Lévy search, $T \simeq D_{\mathrm{L}}^{-1 / \alpha}$, whereas $T \simeq D_{\mathrm{L}}^{-1 /(2-\alpha)}$ in the sloppy Lévy search, where $\alpha<1$. The Lévy component is thus taken most profit of when $\alpha$ approaches 1. Generally, too short jumps, leading to local oversampling, as well as too long jumps, missing the target, are unfavorable.

A crucial assumption of the model, analogous to the derivation in Ref. [6], is that on the time scale of the diffusion process the polymer chain appears annealed; otherwise, individual jumps are no longer uncorrelated [5]. Generally, for proteins $D_{\mathrm{B}}$ is fairly low, and can be further lowered by adjusting the salt condition, so that the conditions for the annealed case can be met. Conversely, by increasing $D_{\mathrm{B}}$ in respect to the polymer dynamics, leading to a higher probability to use the same loopinginduced 'shortcut' repeatedly, it might be possible to investigate the turnover from LF motion to 'paradoxical diffusion' of the quenched polymer case [5].

Single molecule studies can probe the dynamics of the target search and the quantitative predictions of our model [10, 17]. Monitoring the target finding dynam- ics may also be a novel way of investigating soft matter properties regarding both polymer equilibrium configurations, giving rise to $\alpha$, and its dynamics. With respect to the first arrival properties, it would be interesting to study the gradual change of the polymer properties from self-avoiding behavior in a good solvent to Gaussian chain statistics under $\theta$ or dense conditions.

In a next step, it will be of interest to explore effects on the DNA looping behavior due to (a) the occurrence of local denaturation bubbles performing as hinges [18], whose dynamics can be understood from statistical approaches [19]; or (b) kinks imprinted on the DNA locally by binding proteins. In the presence of different protein species, the first arrival method may provide a way to probe protein crowding effects to expand existing models toward the in vivo situation.

Conclusion. Our search model reveals rich behavior in dependence of the LF diffusivity $D_{\mathrm{L}}$ and exponent $\alpha$. In particular, we found two crossovers for the optimal search that we expect to be accessible experimentally. In that sense, our model system is richer than the $2 \mathrm{D}$ albatross search model [4]. We note that in the Cauchy case $\alpha=1$ additional logarithmic contributions are superimposed to the power laws [20]. Moreover, long-time memory effects may occur in the process; in the protein search, e.g., there are indications that both the sliding search through stronger protein-DNA interactions $[9]$ and the volume diffusion through crowding effects are subdiffusive [3].

We thank I. M. Sokolov and U. Gerland for discussions.
[1] B. D. Hughes Random Walks and Random Environments (Oxford University Press, Oxford, 1995), Vol. 1.

[2] P. Lévy, Théorie de l'addition des variables aléatoires (Gauthier-Villars, Paris, 1954).

[3] R. Metzler and J. Klafter, Phys. Rep. 339, 1 (2000); J. Phys. A 37, R161 (2004).

[4] G. M. Viswanathan et al., Nature 401, 911 (1999).

[5] I. M. Sokolov, J. Mai and A. Blumen, Phys. Rev. Lett. 79, 857 (1997).

[6] D. Brockmann and T. Geisel, Phys. Rev. Lett. 91, 048303 (2003).

[7] B. Duplantier, J. Stat. Phys. 54, 581 (1989).

[8] A. Bakk and R. Metzler, FEBS Lett. 563, 66 (2004); J. Theor. Biol. 231, 525 (2004), and references. therein.

[9] M. Slutsky and L. A. Mirny, Biophys. J. 87, 4021 (2004).

[10] I. M. Sokolov, R. Metzler, K. Pant, and M. C. Williams, Biophys. J. 89, 895 (2005); Phys. Rev. E 72, 041102 (2005).

[11] O. G. Berg, R. B. Winter, and P. H. von Hippel, Biochem. 20, 6929 (1981).

[12] A. V. Chechkin et al., J. Phys. A 36, L537 (2003).

[13] For identical proteins, their mutual avoidance is actually included in Eq. (1), as on encounter it does not matter whether they deflect each other or swap identities.

[14] Note that the dimension of the on and off rates differ; while $\left[k_{\text {off }}\right]=\mathrm{sec}^{-1}$, we chose $\left[k_{\text {on }}\right]=\mathrm{cm}^{2} / \mathrm{sec}$.

[15] The symbol $\sim$ implies that the relative difference vanishes, e.g.: $\left.\lim _{k_{\text {off }}+u \rightarrow \infty} W_{0}(u)\right|_{D_{\mathrm{L}}=0} / W_{0}(u)=1$.

[16] M. Coppey, O. Bénichou, R. Voituriez, and M. Moreau, Biophys. J. 87, 1640 (2004).

[17] R. Metzler and T. Ambjörnsson, J. Comp. Theor. Nanoscience 2, 389 (2005), and references therein.

[18] J. Yan and J. F. Marko, Phys. Rev. Lett. 93, 108108 (2004).

[19] T. Ambjörnsson and R. Metzler, Phys. Rev. E 72, 030901(R) (2005); S. K. Banik, T. Ambjörnsson, and R. Metzler, Europhys. Lett. 71, 852 (2005).

[20] For $\alpha=1$ and $k_{\mathrm{off}}+u \ll \tau_{\mathrm{BL}}^{-1}$ we find $W_{0}(u) \sim$ $\left(\pi D_{\mathrm{L}}\right)^{-1} \log \left\{1 /\left[\tau_{\mathrm{BL}}\left(u+k_{\text {off }}\right)\right]\right\}$. At $k_{\text {off }}^{-1} \gg t \gg \tau_{\mathrm{BL}}$, Tauberian theorems [1] lead to the logarithmic correction $J(t) \sim t /\left[\tau_{6} \log \left(t / \tau_{\mathrm{BL}}\right)\right]$, with $\tau_{6}=1 /\left(\pi n_{0} D_{\mathrm{L}}\right)$, while for $t \gg k_{\text {off }}^{-1} \gg \tau_{\mathrm{BL}}$, we have $J(t) \sim t /\left[\tau_{6} \log \left\{1 /\left(\tau_{\mathrm{BL}} k_{\mathrm{off}}\right)\right\}\right]$. 